\title{
The qualifier "primary" should not be used too lightly in the context of measurement
}

\author{
Paul De Bièvre
}

Published online: 18 January 2011

(C) Springer-Verlag 2011

In a context of measurement, the adjective "primary" is frequently used in conjunction with 'measurement standard' [concepts here in the text are denoted in single quotes; double quotes are used to quote from elsewhere or to emphasize]:

- by sellers of 'reference materials' who want to stress the value of their materials by allocating the adjective "primary" to them,

- by National Metrology Institutes (NMIs), who want to declare their measurement standards "primary" so that the customer selects (and buys) them for use in his/her measurement procedures,

- by measurement laboratories who claim this label to convince their customers to buy measurement services from them,

- by scientists in their published papers to convince the reader that they do describe the best measurements possible and, in any case, better than (s)he could find elsewhere.

But what means "primary" in the context of "metrology'? The adjective "primary" is used to characterize material end points in the metrological traceability chain, e.g., whenever a theoretical, and therefore of necessity abstract, definition is "realized" (materialized) for practical use in 'measurement'.

We find the answer in the "International vocabulary of metrology-basic concepts and associated terms" (VIM) [1] where in entry 5.4 we read:

P. De Bièvre $(\square)$

Kasterlee, Belgium

e-mail: paul.de.bievre@skynet.be primary measurement standard

measurement standard established using a primary reference measurement procedure, or created as an artifact, chosen by convention

[in the VIM, terms used for a concept to be defined are printed in bold face. In the text of a given entry, terms of concepts defined elsewhere in the VIM are also printed in bold face the first time they appear].

So, what is a 'measurement standard'? The answer in the VIM is entry 5.1:

\section{measurement standard}

realization of a given quantity, with stated quantity value and associated measurement uncertainty, used as a reference

And what is a primary reference measurement procedure? Again, the answer is given in the VIM under entry 2.9:

primary reference measurement procedure reference measurement procedure used to obtain a measurement result without relation to a measurement standard for a quantity of the same kind

If we would know what is a 'reference measurement procedure', we could go back to our first question: when can we use the qualifier "primary"? Once more, we consult the VIM (entry 2.7 in [1]):

reference measurement procedure measurement procedure accepted as providing measurement results fit for their intended use in assessing measurement trueness of measured quantity values obtained from other 'measurement procedures' for quantities of the same kind, in calibration, or in characterizing reference materials 
Finally, we consult the VIM to know what is a 'measurement procedure' and we find it in entry 2.6:

\section{measurement procedure}

detailed description of a measurement according to one or more measurement principles and to a given measurement method, based on a measurement model and including any calculation to obtain a measurement result

Now we have the conceptual and terminological instrumentarium to find the answer to our first question.

We start with 'measurement procedure' and note that it is a detailed description of a measurement, meaning much more than just a 'measurement method' (entry 2.5 in [1]) because it involves the way the measurement must be carried out by the analyst. The definition contains a condition which must be fulfilled by a "measurement procedure' to merit the adjectivally used noun "reference". Building on that we can designate a 'reference measurement procedure' (entry 2.7 in [1]). Note that the idea of 'reference' in 'metrology' must always be based on an agreement amongst the analysts concerned. Now we get to the stage that we can decide on the nomination of a 'primary reference measurement procedure'. Apart from the requirements put on a 'measurement procedure' and of a 'reference measurement procedure', the quantity value resulting from the use of a 'primary reference measurement procedure' must have been obtained without using a 'measurement standard' for the same kind of quantity. One cannot use 'measurement standards' (and 'measurement procedures') for which the 'primary reference measurement procedure' is intended to serve as reference. The wording of the definition prevents circular reasoning which would be self-defeating because self-fulfilling.

We continue: a 'primary measurement standard' is a special case of 'measurement standard' i.e. one which is only realized through the use of a 'primary reference measurement procedure'. A simple example illustrates that: the certified 'quantity value' in a 'primary measurement standard' for measurements of glucose in water cannot be established by the same 'measurement procedures' which are used in practice for the measurement of glucose in water. The reason is that these 'measurement procedures' need a 'primary measurement standard' which must therefore be given its authority from elsewhere to enable it to be "primary". The certified 'quantity value' embodied in a 'primary measurement standard' must be established using other procedures, for example dissolving a known (e.g. weighed) amount of glucose in a known (e.g. weighed) amount of water (and homogeneizing the resulting solution-of course).

Use of defined metrological concepts preclude "circularity" in the metro-logical and chrono-logical sequence of thinking and acting in the operation 'measurement'.

They must make things simpler and straightforward, not more complicated as is often the case in practical measurement on the laboratory floor.

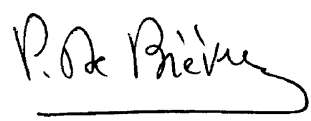

Paul De Bièvre

Editor-in-Chief

\section{By the way, looking for justification of Metrology in Chemistry?}

Sri Lanka tea export, worth $90 \cdot 10^{9}$ Rps (USD $0.8 \cdot 10^{9}$ ) in 2008, was hampered by lack of measurement capability for pesticides and international recognition of the seller's measurement results.

Source: Ministers responsible for Export Development and International Trade, and the Sri Lanka Tea Board and Planters' Association of Ceylon [quoted by R Kaarls at the 5th Brasilian Congress of Metrology, 9 September 2009 in Salvador, Bahia, Brasil].

\section{Reference}

1. BIPM, IEC, IFCC, ILAC, ISO, IUPAC, IUPAP, OIML (2008) International vocabulary of metrology-Basic and general concepts and associated terms (VIM), edition 3, JCGM 200:2008. http://www.bipm.org/vim 